\title{
The southern molecular environment of SNR G18.8+0.3 ${ }^{\star}$ (Research Note)
}

\author{
S. Paron ${ }^{1,2,3}$, M. Celis Peña ${ }^{1}$, M. E. Ortega ${ }^{1}$, A. Petriella ${ }^{1,3}$, M. Rubio ${ }^{4}$, G. Dubner ${ }^{1}$, and E. Giacani ${ }^{1,2}$ \\ ${ }^{1}$ Instituto de Astronomía y Física del Espacio (IAFE), CC 67, Suc. 28, 1428 Buenos Aires, Argentina \\ e-mail: sparon@iafe.uba.ar \\ 2 FADU - Universidad de Buenos Aires, Ciudad Universitaria, C1428EGA Buenos Aires, Argentina \\ 3 CBC - Universidad de Buenos Aires, Ciudad Universitaria, 1053 Buenos Aires, Argentina \\ ${ }^{4}$ Departamento de Astronomía, Universidad de Chile, Casilla 36-D, Santiago, Chile
}

Received 8 May 2015 / Accepted 3 July 2015

\begin{abstract}
Aims. In a previous paper we investigated the molecular environment toward the eastern border of the supernova remnant (SNR) G18.8+0.3. Continuing with the study of the surroundings of this SNR, we now focus on its southern border, which in the radio continuum emission shows a very peculiar morphology with a corrugated corner and a very flattened southern flank.

Methods. We observed two regions toward the south of SNR G18.8+0.3 using the Atacama Submillimeter Telescope Experiment (ASTE) in the ${ }^{12} \mathrm{CO} J=3-2$. One of these regions was also surveyed in ${ }^{13} \mathrm{CO}$ and $\mathrm{C}^{18} \mathrm{O} J=3-2$. The angular and spectral resolution of these observations were $22^{\prime \prime}$ and $0.11 \mathrm{~km} \mathrm{~s}^{-1}$. We compared the $\mathrm{CO}$ emission to $20 \mathrm{~cm}$ radio continuum maps obtained as part of the Multi-Array Galactic Plane Imaging Survey (MAGPIS) and $870 \mu \mathrm{m}$ dust emission extracted from the APEX Telescope Large Area Survey of the Galaxy.

Results. We discovered a molecular feature with a good morphological correspondence with the SNR's southernmost corner. In particular, there are indentations in the radio continuum map that are complemented by protrusions in the molecular $\mathrm{CO}$ image, strongly suggesting that the SNR shock is interacting with a molecular cloud. Toward this region we find that the ${ }^{12} \mathrm{CO}$ peak is not correlated with the observed ${ }^{13} \mathrm{CO}$ peaks, which are likely to be related to a nearby HII region. Regarding the most flattened border of SNR G18.8+0.3, where an interaction of the SNR with dense material was previously suggested, our ${ }^{12} \mathrm{CO} J=3-2$ map shows no obvious indication that this is occurring.
\end{abstract}

Key words. ISM: clouds - ISM: supernova remnants - HII regions

\section{Introduction}

The investigation of the surroundings of supernova remnants (SNRs) can provide useful information for a wide range of research fields, such as the physical and chemical changes induced by the passage of a shock wave, dust production and destruction, the possibility of triggering stellar formation, and production of high-energy gamma rays. Multiwavelength studies are required to explore these effects.

With its peculiar shape with flattened borders along the east and south, it has been suggested that the SNR G18.8+0.3 is interacting with dense molecular gas in these directions (Dubner et al. 1999, 2004; Tian et al. 2007). Paron et al. (2012; hereafter Paper I) investigated the molecular gas toward the eastern border of the SNR G18.8+0.3. They found a dense molecular clump close to the shock front of the SNR but not in contact with it, containing a complex of HII regions and massive young stellar objects (YSOs) that are deeply embedded. This region is indicated in Fig. 1 as "Eastern Clump". Figure 1 shows the SNR G18.8+0.3 in radio continuum at $20 \mathrm{~cm}$ as extracted from the MAGPIS Helfand et al. (2006; in blue) and its surroundings as seen at the $870 \mu \mathrm{m}$ emission extracted from the ATLASGAL Beuther et al. (2012; in green). Southwards of the SNR, where the molecular cloud appears to be in contact with SNR shock front (see Fig. 1 in PaperI), there are a few HII regions and

\footnotetext{
* The data cubes are only available at the CDS via anonymous ftp to cdsarc.u-strasbg.fr (130.79.128.5) or via http://cdsarc.u-strasbg.fr/viz-bin/qcat?]/A+A/580/A51
}

submillimeter dust compact sources, making this region an interesting target for studying the interplay of the SNR shock front, surrounding molecular gas, and HII regions.

Using molecular lines we investigate two regions toward the south of SNR G18.8+0.3; Reg. 1 and the subregion Reg. 1b, were selected to investigate the possible causes of the peculiar morphology of the SNR southernmost "corner" and the molecular gas toward the HII region G018.630+0.309 (Anderson et al. 2011), and the ATLASGAL source 018.626+00.297 (Urquhart et al. 2014), while Reg. 2 was chosen to investigate the molecular environment toward the most flattened border of the SNR where originally it was suggested that there is an interaction with a dense cloud (Dubner et al. 1999). As in Paper I, here we assume a distance of $14 \pm 1 \mathrm{kpc}$ for SNR G18.8+0.3. For the HII region G018.630+0.309, Anderson et al. (2012) could not resolve the distance ambiguity between 1.5 and $14.7 \mathrm{kpc}$.

\section{Observations}

The observations of the molecular lines were carried out between September 7 and 13, 2013 with the $10 \mathrm{~m}$ Atacama Submillimeter Telescope Experiment (ASTE; Ezawa et al. 2004). We used the CATS345 receiver, which is a two-single band SIS receiver remotely tunable in the LO frequency range of $324-372 \mathrm{GHz}$. The surveyed regions are shown in Fig. 1 and summarized in Table 1. The observations were performed in position-switching mode, and the mapping grid spacing was $20^{\prime \prime}$ in all cases. The ${ }^{12} \mathrm{CO} J=$ 3-2 line $(345.796 \mathrm{GHz})$ was observed towars Regs. 1 and 2. In 


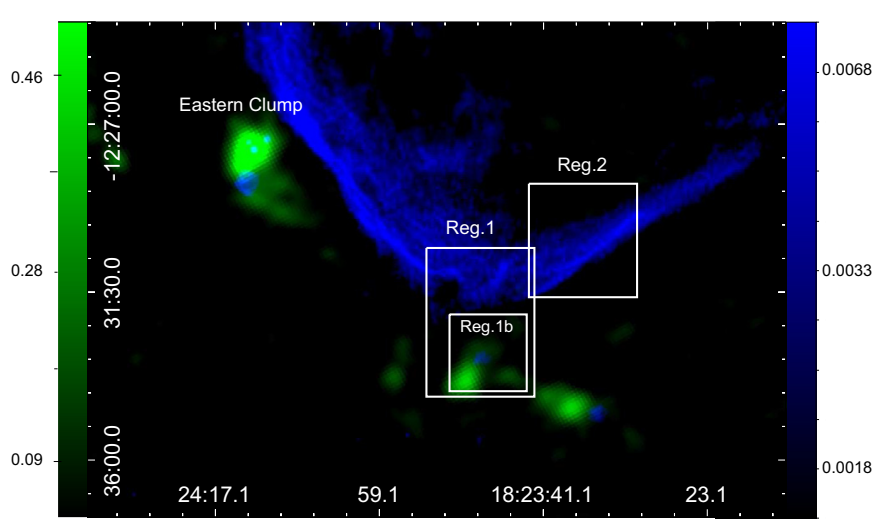

Fig. 1. Two-color image of the SNR G18.8+0.3 and its surroundings. The radio continuum emission at $20 \mathrm{~cm}$ (from MAGPIS) is displayed in blue (right color bar) and the ATLASGAL emission at $870 \mu \mathrm{m}$ in green (left color bar). Both color ranges are in Jy beam ${ }^{-1}$. The surveyed regions in the molecular lines using ASTE are indicated.

Table 1. Surveyed regions using ASTE.

\begin{tabular}{lcc}
\hline \hline Region & Center $(\mathrm{RA}, \mathrm{Dec})$ & Size \\
\hline Reg. 1 & $18: 23: 48.0,-12: 32: 14.5$ & $3^{\prime} \times 4^{\prime}$ \\
Reg. 1b & $18: 23: 47.1,-12: 33: 12.1$ & $2^{\prime} \times 2^{\prime}$ \\
Reg. 2 & $18: 23: 38.0,-12: 30: 04.6$ & $3^{\prime} \times 3^{\prime}$ \\
\hline
\end{tabular}

Reg. 1b, we also observed $\mathrm{C}^{18} \mathrm{O} J=3-2(329.330 \mathrm{GHz})$, and ${ }^{13} \mathrm{CO} J=3-2(330.587 \mathrm{GHz})$. The integration time was $20 \mathrm{~s}$ for the ${ }^{12} \mathrm{CO}$ and $50 \mathrm{~s}$ for the other molecular lines in each pointing.

We used the XF digital spectrometer with bandwidth and spectral resolution set to $128 \mathrm{MHz}$ and $125 \mathrm{kHz}$, respectively. The velocity resolution was $0.11 \mathrm{~km} \mathrm{~s}^{-1}$, the half-power beamwidth (HPBW) 22", and the main beam efficiency $\eta_{\mathrm{mb}} \sim$ 0.65 . The spectra were Hanning-smoothed to improve the signalto-noise ratio and only linear or some second-order polynomia were used for baseline fitting. The data were reduced with NEWSTAR $^{1}$, and the spectra processed using the XSpec software package ${ }^{2}$.

\section{Results and discussion}

\subsection{Regions 1 and $1 b$}

In Reg. $1,{ }^{12} \mathrm{CO} J=3-2$ emission is detected at velocities between 5 and $30 \mathrm{~km} \mathrm{~s}^{-1}$, very much like what was observed in the eastern region (Paper I). The emission integrated over the mentioned velocity range is displayed as contours over a twocolor image showing the $20 \mathrm{~cm}$ and $870 \mu \mathrm{m}$ continuum images in Fig. 2. Important is that the boundaries of the radio continuum emission from the SNR and the ${ }^{12} \mathrm{CO}$ emission from the molecular cloud show several common features, strongly suggesting that the shock front is interacting with the cloud. In particular, the most noticeable indentations on the border of the SNR are complemented by protrusions in the molecular cloud. In addition, toward the south the contour at $56 \mathrm{~K} \mathrm{~km} \mathrm{~s}^{-1}$ of the ${ }^{12} \mathrm{CO}$ emission has a peculiar curvature apparently coinciding with the HII region G018.630+0.309, suggesting that this object is also associated with the molecular structure.

\footnotetext{
1 Reduction software based on AIPS developed at NRAO and extended to treat single dish data with a graphical user interface (GUI).

2 XSpec is a spectral line reduction package for astronomy that has been developed by Per Bergman at Onsala Space Observatory.
}

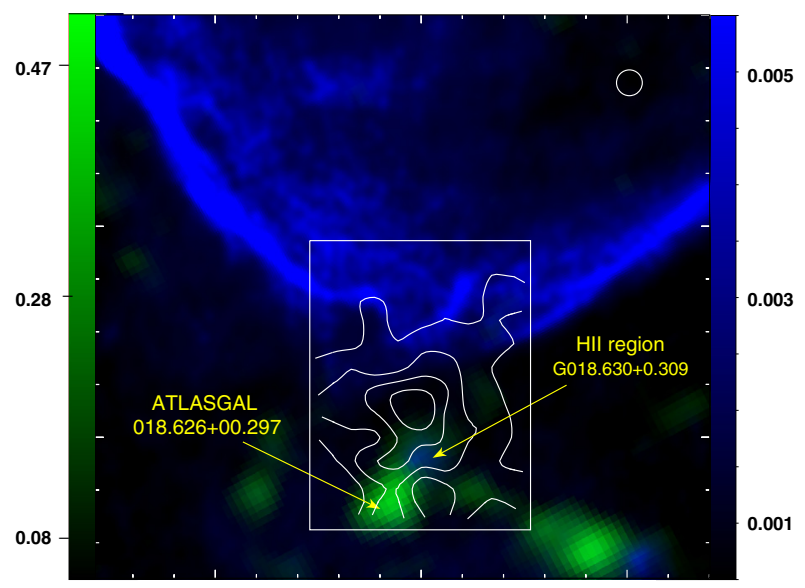

Fig. 2. Two-color image toward Reg. 1 with the radio continuum emission at $20 \mathrm{~cm}$ (from MAGPIS) displayed in blue (right color bar) and the ATLASGAL emission at $870 \mu \mathrm{m}$ in green (left color bar). Both color ranges are in $\mathrm{Jy}$ beam $^{-1}$. The ${ }^{12} \mathrm{CO} J=3-2$ emission integrated between 5 and $30 \mathrm{~km} \mathrm{~s}^{-1}$ is presented in contours with levels of $32,42,56$, and $68 \mathrm{~K} \mathrm{~km} \mathrm{~s}^{-1}$. The beam of the molecular observations is shown in the top right corner. The positions of the HII region G018.630+0.309 and the compact dust source ATLASGAL 018.626+00.297 are indicated.

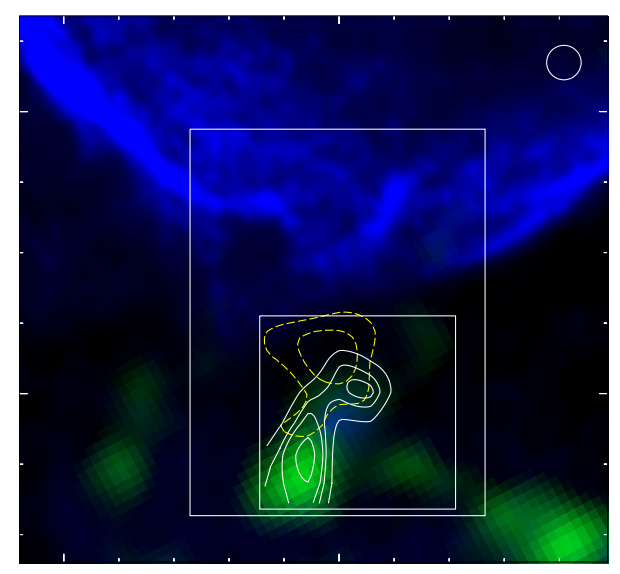

Fig. 3. Two-color image toward Reg. 1b with the radio continuum emission at $20 \mathrm{~cm}$ (from MAGPIS) displayed in blue and the ATLASGAL emission at $870 \mu \mathrm{m}$ in green. The color ranges are the same as in Fig. 2. For context, the box of Reg. 1 and the 56 , and $68 \mathrm{~K} \mathrm{~km} \mathrm{~s}^{-1}$ contours (in dashed yellow line) of the ${ }^{12} \mathrm{CO}$ emission presented in Fig. 2 are also shown. Contours of the ${ }^{13} \mathrm{CO} J=3-2$ emission integrated between 15 and $26 \mathrm{~km} \mathrm{~s}^{-1}$ are shown in white, with levels of 12.0, 15.0, 16.5, and $20.0 \mathrm{~K} \mathrm{~km} \mathrm{~s}^{-1}$. The beam of the molecular observations is shown in the top right corner.

Figure 3 displays the ${ }^{13} \mathrm{CO} J=3-2$ emission integrated between 15 and $26 \mathrm{~km} \mathrm{~s}^{-1}$ toward Reg. $1 \mathrm{~b}$ in white contours. For context, the 56 and $68 \mathrm{~K} \mathrm{~km} \mathrm{~s}^{-1}$ contours of the ${ }^{12} \mathrm{CO}$ emission shown in Fig. 2 are also shown in Fig. 3 (yellow dashed contours). The ${ }^{13} \mathrm{CO}$ emission reveals a molecular feature composed of two clumps that partially surrounds the HII region $\mathrm{G} 018.630+0.309$. One of these clumps, the southernmost one, exactly overlaps the source ATLASGAL 018.626+00.297. Curiously the most intense emission of the ${ }^{12} \mathrm{CO}$ is not correlated with the ${ }^{13} \mathrm{CO}$ peaks. For the $\mathrm{C}^{18} \mathrm{O} J=3-2$ line, we only detect emission (a single spectrum) over the maximum of the ATLASGAL source, implying that this is the region with highest density. 
To discuss the molecular gas in Regs. 1 and $1 \mathrm{~b}$, we denote the structure delimited by the $56 \mathrm{~K} \mathrm{~km} \mathrm{~s}^{-1}$ contour (the dashed yellow contour in Fig. 3) as the "12CO-clump". The feature delimited by a circle of $25^{\prime \prime}$ in radius centered on the northern maximum in the ${ }^{13} \mathrm{CO}$ emission is designated as the "13 CO-clumpN" and the southern ${ }^{13} \mathrm{CO}$ clump delimited by the $16.5 \mathrm{~K} \mathrm{~km} \mathrm{~s}^{-1}$ contour as the "13 CO-clumpS" (see Fig. 3). We estimated the mass of these features by applying a variety of methods: by assuming local thermodynamic equilibrium (LTE), from the CO luminosity (in the case of the ${ }^{12} \mathrm{CO}$-clump), from the thermal dust emission at $870 \mu \mathrm{m}$ (in the case of the ${ }^{13} \mathrm{CO}-$ clumpS), and from the virial theorem.

To obtain the LTE mass, we followed the procedures described in Paper I to estimate the column densities of $N\left({ }^{12} \mathrm{CO}\right)$ and $N\left({ }^{13} \mathrm{CO}\right)$. Table 2 presents the derived and used parameters to do that. The column density values represent the total column density as obtained from summation over all beam positions belonging to each molecular structure described above. To obtain the mass from the $\mathrm{CO}$ luminosity, we used (e.g., Bertsch et al. 1993): $N\left(\mathrm{H}_{2}\right)=2 \times 10^{20} W\left({ }^{12} \mathrm{CO}\right)$ to estimate $N\left(\mathrm{H}_{2}\right)$, where $W\left({ }^{12} \mathrm{CO}\right)$ is the velocity-integrated ${ }^{12} \mathrm{CO}$ emission throughout the ${ }^{12} \mathrm{CO}$-clump structure. Finally, once the total $N\left(\mathrm{H}_{2}\right)$ has been obtained, either from the LTE approximation or from the CO luminosity, the mass was derived using $M=$ $\mu m_{\mathrm{H}} d^{2} \Omega N\left(\mathrm{H}_{2}\right)$, where $\Omega$ is the solid angle subtended by the beam size, $m_{\mathrm{H}}$ the hydrogen mass, $d$ the distance, $\mu$ the mean molecular weight, assumed to be 2.8 by taking a relative helium abundance of $25 \%$ into account.

Assuming that the ${ }^{13} \mathrm{CO}$-clumpS is related to the ATLASGAL source 018.626+00.297, we estimated its mass from the thermal dust emission at $870 \mu \mathrm{m}$ following the procedure presented in Csengeri et al. (2014). We used an integrated flux of $S_{\text {int }}=3.31 \mathrm{Jy}$ (Urquhart et al. 2014) and assumed a typical dust temperature of $T_{\mathrm{d}}=15 \mathrm{~K}$.

Finally, the virial mass of the three molecular structures was derived from the relation:

$M_{\mathrm{vir}}=\frac{5 R_{\mathrm{clump}}(\Delta \mathrm{v})^{2}}{8 G \ln (2)}$

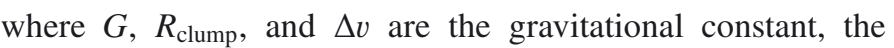
clump radius (assuming a spherical geometry), and the line width, respectively. Following Saito et al. (2007), we defined the clump radius as the deconvolved radius calculated from $R_{\text {clump }}=\sqrt{\frac{S-\text { beam area }}{\pi}}$, where $S$ is the area inside the clump. The velocity width $\Delta v$ was derived through a Gaussian fitting to the averaged spectrum obtained for the molecular features. The deconvolved radius $\left(R_{\text {clump }}\right)$ are $2.95,1.52$, and $1.05 \mathrm{pc}$, and the $\Delta v=7.05,3.80$, and $5.10 \mathrm{~km} \mathrm{~s}^{-1}$ for the ${ }^{12} \mathrm{CO}$-clump, ${ }^{13} \mathrm{CO}-$ clumpN, and ${ }^{13} \mathrm{CO}$-clumpS, respectively. All mass results are presented in Table 3.

The only case in which the virial mass differs considerably from the mass obtained by other methods is for the ${ }^{12} \mathrm{CO}$-clump. For this feature, the virial mass is almost an order of magnitude higher than the LTE and CO luminosity-based estimates of the mass. This suggests that this feature is not gravitationally bound.

As a working hypothesis we assume that the ${ }^{12} \mathrm{CO}$-clump is a small molecular feature supported by external pressure. The external pressure $\left(P_{\text {ext }}\right)$ required for the ${ }^{12} \mathrm{CO}$ clump to remain bound, can be roughly evaluated from the following expression of the virial theorem (e.g., Kawamura et al. 1998):

$2 U+\Omega-4 \pi R_{\text {clump }}{ }^{3} P_{\text {ext }}=0$
Table 2. Used and derived parameters for the LTE mass.

\begin{tabular}{lccc}
\hline \hline Parameter & ${ }^{12} \mathrm{CO}$-clump & ${ }^{13} \mathrm{CO}$-clumpN & ${ }^{13} \mathrm{CO}$-clumpS \\
\hline$T_{\text {ex }}(\mathrm{K})$ & 16.6 & 12.5 & 11.0 \\
$\tau^{12}$ & 15 & - & - \\
$\tau^{13}$ & - & 1.3 & 3.5 \\
$N\left({ }^{12} \mathrm{CO}\right)\left(\mathrm{cm}^{-2}\right)^{*}$ & $7.1 \times 10^{18}$ & - & - \\
$N\left({ }^{13} \mathrm{CO}\right)\left(\mathrm{cm}^{-2}\right)^{\dagger}$ & - & $1.5 \times 10^{17}$ & $3.5 \times 10^{17}$ \\
$N\left(\mathrm{H}_{2}\right)\left(\mathrm{cm}^{-2}\right)$ & $7.1 \times 10^{22}$ & $7.5 \times 10^{22}$ & $1.7 \times 10^{23}$ \\
\hline
\end{tabular}

Notes. ${ }^{(*)}$ To obtain the $N\left(\mathrm{H}_{2}\right)$ from the $N\left({ }^{12} \mathrm{CO}\right)$ we assume the canonical abundance ratio $\left[{ }^{12} \mathrm{CO} / \mathrm{H}_{2}\right]=10^{-4}$. (†) To obtain the $N\left(\mathrm{H}_{2}\right)$ from the $\mathrm{N}\left({ }^{13} \mathrm{CO}\right)$ we assume $\left[{ }^{13} \mathrm{CO} / \mathrm{H}_{2}\right]=2 \times 10^{-6}$ (e.g. Simon et al. 2001).

Table 3. Mass values.

\begin{tabular}{lcccc}
\hline \hline Clump & $\begin{array}{c}M_{\mathrm{LTE}} \\
\left(M_{\odot}\right)\end{array}$ & $\begin{array}{c}M_{\mathrm{CO} \text { lum. }} \\
\left(M_{\odot}\right)\end{array}$ & $\begin{array}{c}M_{\text {dust }} \\
\left(M_{\odot}\right)\end{array}$ & $\begin{array}{c}M_{\text {vir }} \\
\left(M_{\odot}\right)\end{array}$ \\
\hline${ }^{12}$ CO-clump & $2.7 \times 10^{3}$ & $6.0 \times 10^{3}$ & - & $3.1 \times 10^{4}$ \\
${ }^{13} \mathrm{CO}-$ clumpN & $3.0 \times 10^{3}$ & - & - & $4.5 \times 10^{3}$ \\
${ }^{13} \mathrm{CO}-$ clumpS & $7.0 \times 10^{3}$ & - & $5.5 \times 10^{3}$ & $5.7 \times 10^{3}$ \\
\hline
\end{tabular}

where

$U=3 / 2 M \frac{\Delta v^{2}}{8 \ln (2)}$, and $\Omega=-\frac{3 G M^{2}}{5 R_{\text {clump }}}$.

Using a mean value between $M_{\mathrm{LTE}}$ and $M_{\mathrm{CO}}$ lum. for the mass, we found that a $P_{\text {ext }} / k_{\mathrm{B}} \sim 1.4 \times 10^{6} \mathrm{~cm}^{-3} \mathrm{~K}$, with $k_{\mathrm{B}}$ the Boltzmann constant, is required to keep the ${ }^{12} \mathrm{CO}$-clump bound. This value is at least one order of magnitude greater than the external pressures calculated in Kawamura et al. (1998) for a sample of clouds, where it is considered that the pressure is produced by the surrounding diffuse ambient gas. Thus, if this clump is supported by external pressure, it is required a pressure source, which in the present context is likely to be the SNR shocks running into the outer layers of the molecular gas. The derived $P_{\text {ext }} / k_{\mathrm{B}}$ is very similar to the pressure obtained toward a region associated with the face-on shock in the Vela SNR using FUSE observations (Sankrit et al. 2001), and this provides further support to our hypothesis that a SNR shock front contributes to maintaining the observed ${ }^{12} \mathrm{CO}$-clump as bound.

Finally we perform a SED analysis of the IR counterpart of HII region G018.630+0.30, the WISE source J182348.08123316.1 (Cutri \& et al. 2013; Wright et al. 2010). The fitting of the SED was done using the tool developed by Robitaille et al. $(2007)^{3}$ that considers the fluxes at the WISE 3.4, 4.6, 12, and $22 \mu \mathrm{m}$ bands, an $A_{v}$ between 14 and $50 \mathrm{mag}$ (see Paper I) and a distance range of $13-15 \mathrm{kpc}$. The SED results show that the source is indeed a young massive star (age $\sim 10^{5} \mathrm{yrs}$ and mass $\sim 12 M_{\odot}$ ), suggesting that its formation should be approximately coeval with the SN explosion. If the SED is performed using the near distance (a range 1-3 kpc), we find that the source should have a lower mass (about $3 M_{\odot}$ ), which would be unable to ionize the gas and generate an HII region. This is an indirect confirmation that the HII region are located at the farther distance, in turn suggesting that it should be embedded in the molecular cloud southward of the SNR.

http://caravan.astro.wisc.edu/protostars/ 
A\&A 580, A51 (2015)
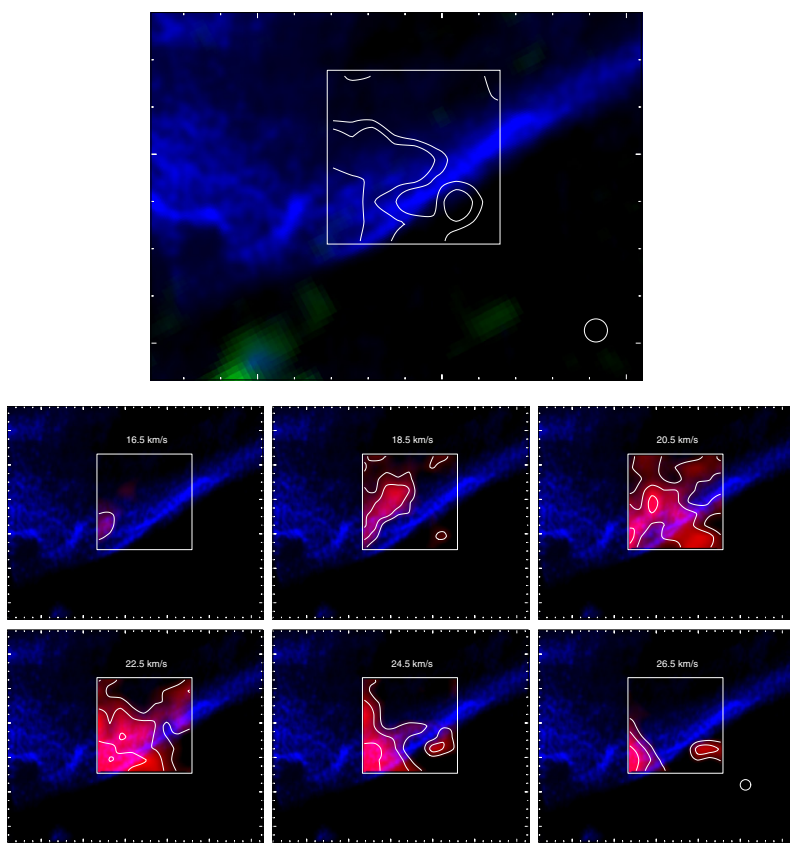

Fig. 4. Upper: ${ }^{12} \mathrm{CO} J=3-2$ emission integrated between 15 and $30 \mathrm{~km} \mathrm{~s}^{-1}$, with levels of 14,18 , and $28 \mathrm{~K} \mathrm{~km} \mathrm{~s}^{-1}$ (white contours) toward Reg. 2. The color ranges are the same as in Fig. 2. Bottom: channel maps of the ${ }^{12} \mathrm{CO} J=3-2$ emission (red with white contours) integrated in steps of $2 \mathrm{~km} \mathrm{~s}^{-1}$. The contours levels are 2.5, 4.0, and $6.0 \mathrm{~K} \mathrm{~km} \mathrm{~s}^{-1}$.

\subsection{Region 2}

The ${ }^{12} \mathrm{CO}$ emission from this region is significantly weaker than was detected toward Reg. 1. By integrating the ${ }^{12} \mathrm{CO} \mathrm{J}=$ 3-2 emission between 15 and $30 \mathrm{~km} \mathrm{~s}^{-1}$ (see Fig. 4 upper), which is the whole velocity range where there is emission, we do not find any conspicuous morphological correspondence between the molecular gas and the most flattened border of the SNR, which might suggest some kind of interaction. To appreciate the behavior of the molecular gas in this region in more detail, we present the ${ }^{12} \mathrm{CO}$ emission in a series of channel maps integrated in steps of $2 \mathrm{~km} \mathrm{~s}^{-1}$ at the bottom of Fig. 4. It can be seen that the molecular gas has a very clumpy distribution not only on the plane of the sky but also along the line of sight. The ${ }^{12} \mathrm{CO}$ emission shown in the three first panels in Fig. 4 (bottom) may correspond to gas located in front (along the line of sight) of the SNR G18.9+0.3, while the emission displayed in the remaining panels would suggest a correspondence between the SNR and the molecular gas (see mainly the small clump toward the west in panel 24.5 and $26.5 \mathrm{~km} \mathrm{~s}^{-1}$ ). We note that the molecular emission toward the bottom lefthand corner of the surveyed region, mainly at $22.5 \mathrm{~km} \mathrm{~s}^{-1}$, should belong to the upper righthand border of the structure analyzed in Reg. 1. Thus, even though the gas distribution in the last panels of Fig. 4 (bottom) may suggest a correspondence between the SNR and the molecular gas, these results allow us to conclude that the unusual flattened border of SNR G18.8+0.3 must have some physical origin other than interaction with dense environmental gas.

\section{Summary and concluding remarks}

We report the analysis of the molecular gas toward the southern environment of the SNR G18.8+0.3 using ASTE observations. We surveyed two regions, one toward the SNR's southernmost "corner", which in addition harbors the HII region
G018.630+0.309, and a second one toward the most flattened border of the SNR. The main results can be summarized as follows.

(a) In the first region, named Reg. 1/Reg. 1b, we discovered a molecular feature with a good morphological correspondence with the SNR southernmost corner, where the indentations in the morphology of the SNR radio continuum emission are complemented by protrusions in the molecular cloud, strongly suggesting a SNR-molecular cloud interaction. Additionally, analyzing this molecular cloud with the ${ }^{13} \mathrm{CO} J=3-2$ line, we found a molecular feature composed of two clumps that are not correlated to the ${ }^{12} \mathrm{CO}$ peak, which partially surrounds the HII region G018.630+0.309 and correlates with the thermal dust emission at $870 \mu \mathrm{m}$. We found that the virial mass of the ${ }^{12} \mathrm{CO}$-clump differs considerably from the mass value obtained by other methods, suggesting that it is not gravitationally bound. Finally, from a SED fitting to the IR counterpart of the HII region, we confirm that it is located at the far distance, suggesting that it should be embedded in the molecular cloud southward of the SNR.

(b) The second region, named Reg. 2, covers a portion of the most flattened border of SNR G18.8+0.3, where it was originally suggested that there is an interaction with a dense cloud (Dubner et al. 1999). Strikingly, our ${ }^{12} \mathrm{CO} J=3-2$ observations show a clumpy molecular structure without any morphological correspondence with the flat border of the SNR that could suggest a causal relationship. The better angular resolution attained in these new data served to reveal that the flat southern border of the SNR must originate in a different process than the compression of a dense molecular cloud.

Acknowledgements. The ASTE project is led by Nobeyama Radio Observatory (NRO), a branch of National Astronomical Observatory of Japan (NAOJ), in collaboration with University of Chile and with Japanese institutes including University of Tokyo, Nagoya University, Osaka Prefecture University, Ibaraki University, Hokkaido University, and Joetsu University of Education. S.P., M.O., A.P., G.D., and E.G. are members of the Carrera del investigador cientifico of CONICET, Argentina. M.C.P. is a doctoral fellow at CONICET, Argentina. This work was partially supported by Argentina grants awarded by UBA (UBACyT), CONICET, and ANPCYT. M.R. wishes to acknowledge support from CONICYT through FONDECYT grant No. 1140839. A.P. is grateful to the ASTE staff for the support received during the observations.

\section{References}

Anderson, L. D., Bania, T. M., Balser, D. S., \& Rood, R. T. 2011, ApJS, 194, 32 Anderson, L. D., Bania, T. M., Balser, D. S., \& Rood, R. T. 2012, ApJ, 754, 62 Bertsch, D. L., Dame, T. M., Fichtel, C. E., et al. 1993, ApJ, 416, 587 Beuther, H., Tackenberg, J., Linz, H., et al. 2012, ApJ, 747, 43

Csengeri, T., Urquhart, J. S., Schuller, F., et al. 2014, A\&A, 565, A75

Cutri, R. M., et al. 2013, VizieR Online Data Catalog: II/328

Dubner, G., Giacani, E., Reynoso, E., et al. 1999, AJ, 118, 930

Dubner, G., Giacani, E., Reynoso, E., \& Parón, S. 2004, A\&A, 426, 201

Ezawa, H., Kawabe, R., Kohno, K., \& Yamamoto, S. 2004, in SPIE Conf. Ser. 5489, ed. J. M. Oschmann, Jr., 763

Helfand, D. J., Becker, R. H., White, R. L., Fallon, A., \& Tuttle, S. 2006, AJ, 131,2525

Kawamura, A., Onishi, T., Yonekura, Y., et al. 1998, ApJS, 117, 387

Paron, S., Ortega, M. E., Petriella, A., et al. 2012, A\&A, 547, A60

Robitaille, T. P., Whitney, B. A., Indebetouw, R., \& Wood, K. 2007, ApJS, 169, 328

Saito, H., Saito, M., Sunada, K., \& Yonekura, Y. 2007, ApJ, 659, 459

Sankrit, R., Shelton, R. L., Blair, W. P., Sembach, K. R., \& Jenkins, E. B. 2001, ApJ, 549, 416

Simon, R., Jackson, J. M., Clemens, D. P., Bania, T. M., \& Heyer, M. H. 2001, ApJ, 551, 747

Tian, W. W., Leahy, D. A., \& Wang, Q. D. 2007, A\&A, 474, 541

Urquhart, J. S., Csengeri, T., Wyrowski, F., et al. 2014, A\&A, 568, A41

Wright, E. L., Eisenhardt, P. R. M., Mainzer, A. K., et al. 2010, AJ, 140, 1868 